\title{
Le médecin dans la cité grecque
}

\author{
par Huldrych M. Koelbing ${ }^{1}$
}

\section{Résumé}

Dans les villes grecques, comme plus tard à Rome, la responsabilité des médecins était déjà un sujet controversé. La médecine était un métier tout à fait libre: aucune protection légale du médecin bien instruit et honnête, aucune sanction contre les charlatans. Aussi les médecins bien instruits menaient souvent une vie itinérante; mais par la nomination de médecins publics, les cités s'efforçaient de s'assurer les services de médecins de qualité, qui d'ailleurs touchaient des honoraires de la part de leurs clients. Les bons médecins, $c$-à-d. les médecins selon le modèle hippocratique, se voyaient confrontés à la concurrence d'une multitude de praticiens plus ou moins empiriques; d'aucuns faisaient même traiter une partie de leur clientèle par un praticien-esclave.

Quelques petits textes ajoutés tardivement au Corpus Hippocraticum nous laissent entrevoir la vie quotidienne du médecin grec. Depuis Hippocrate ( Prognôstikon») à l'époque hellénistique ("De la Bienséance»), nous constatons une évolution importante quant à la révélation d'un pronostic néfaste: tandis qu'Hippocrate favorise la "révélation massue» (D.Gourévitch), son disciple lointain prend en considération la psychologie du malade et lui cache la vérité accablante tout en la révélant à ses proches. C'est la naissance de ce principe de la double vérité, tant contesté aujourd'hui.

\section{Discussions autour de la responsabilité du médecin}

\begin{abstract}
«La médecine est de toutes les professions la plus noble; et cependant, par l'ignorance et de ceux qui l'exercent et de ceux qui jugent à la légère ces derniers, elle est dès à présent reléguée au dernier rang. Un aussi faux jugement me semble provenir principalement de ce que la profession médicale seule n'est, dans les cités, soumise à aucune autre peine qu'à celle de la déconsidération; or, la déconsidération ne blesse pas des gens qui en vivent. Ces gens ressemblent beaucoup aux figurants qu'on fait paraître dans les tragédies; de même que les figurants ont l'apparence, l'habit et le masque d'acteurs sans être acteurs, de même, parmi les médecins, beaucoup le sont par le titre, bien peu le sont par le fait ${ }^{2} . »$
\end{abstract}

Gesnerus 46 (1989) 29-43 
Ainsi s'exprime un médecin grec, inconnu, du $4^{\mathrm{e}}$ siècle avant J.-C., dans le texte très bref connu sous le nom de «La Loi» et qui contient quelques réflexions sur la formation du bon médecin.

A peu près 400 ans plus tard, le Romain Pline l'Ancien (23-79) s'échauffe à propos des médecins ${ }^{3}$ :

«Aucune loi d'autre part ne punit l'ignorance meurtrière; il n'est pas d'exemple qu'elle ait été châtiée. Les médecins s'instruisent à nos risques et périls, ils poursuivent leurs expériences grâce à des morts, et c'est seulement chez le médecin que l'homicide est assuré de l'impunité totale. Bien plus! On transfère le blâme, on rejette la faute sur l'intempérance du malade, et l'on va jusqu'à incriminer ceux qui ont succombé.»

Ce n'est d'ailleurs pas le seul crime que Pline et ses compatriotes reprochent aux médecins, et surtout aux médecins grecs. Dans un livre récent, Danielle Gourévitch nous présente tout un «roman noir de la médecine», extrait de la littérature romaine ${ }^{4}$.

Il est peu probable que Pline ait lu les écrits hippocratiques; tout au plus y aura-t-il cherché quelques remèdes. Pourtant, pour ce qu'il dit à la fin du texte cité, il aurait pu y trouver une confirmation en consultant le discours «De l'Art», composé vers 400 ou 350 avant J.-C., évidemment par un orateur qui s'était fixé comme but de faire l'éloge inconditionnel de l'art médical ${ }^{5}$. Tous les malades qui guérissent, dit-il, le doivent à l'art médical, et cela même s’ils n'ont pas consulté de médecin. Dans ce cas,

«ils ont guéri en faisant ceci ou cela; or, le choix entre ce qui est bon et ce qui est mauvais implique l'existence de l'art».

Il n'existe pas de guérison spontanée, due à «la fortune», au hasard. Le hasard, to automaton, n'existe pas:

«tout ce qui se fait, se fait par un pourquoi; or, devant le pourquoi, le hasard perd visiblement toute réalité».

Dans la connaissance du «pourquoi» et dans la prévision qui découle de cette connaissance, la médecine prouve et prouvera à tout jamais sa réalité.

D'autre part, si le malade meurt au lieu de recouvrer la santé, ce n'est point la faute de l'art médical ou du médecin:

«De fait, il est beaucoup plus vraisemblable que le malade sera incapable d'obéir aux prescriptions, qu'il ne l'est que le médecin fera de mauvaises prescriptions [...]. N'est il pas bien naturel que l'un prescrive convenablement, mais que l'autre n'ait pas le courage d'obéir et, n'obéissant pas, succombe?» 
Enfin, c'est la pure folie de blâmer les médecins parce qu'ils refusent de traiter des maladies désespérées: On ne saurait demander à l'art qu'il accomplisse des choses pour lesquelles les instruments lui manquent!

On dirait que Pline reprend l'argumentation de cet apologète grec de l'art médical - mais d'un point de vue parfaitement opposé.

Les trois textes que je viens de citer illustrent l'appréciation des médecins grecs par eux-mêmes comme par le public, grec ou romain. Car, c'est aussi de médecins grecs que parle Pline, la science médicale à Rome étant et restant un domaine grec. Au début du $1^{\text {er }}$ siècle avant J.-C., avec Asclépiade de Pruse, en Bithynie (Asie Mineure), la médecine grecque s'était installée à Rome, et encore sous l'Empire, les médecins de qualité étaient presque tous des Grecs. Mais déjà Caton le Censeur (234-149) avait mis en garde son fils contre les médecins grecs ${ }^{6}$ :

«Je te parlerai de ces Grecs, Marc mon fils, en temps et lieu [...]. Je prouverai que c'est une race perverse et indocile, et sache bien qu'un oracle te parle lorsque je dis: le jour où cette nation apportera ses sciences, elle corrompra tout, et ce sera bien pis si elle envoie ses médecins. Ils se sont juré d'exterminer tous les barbares par la médecine et cela même, ils le font moyennant salaire, afin de capter la confiance et de tuer plus à leur aise.»

Pline, en citant ces paroles fortes, se trouve en plein accord avec Caton, et il met en relief le fait que les Romains, pendant 600 ans $a b$ urbe condita, avaient vécu sans médecins. Donc: rejet complet de l'art médical grec, cette belle iatrikê technê, par les Romains Caton et Pline, fiers de leur tradition nationale.

Il va de soi que l'auteur du discours «De arte» n'avait aucune raison de condamner ainsi la médecine. Probablement, ce n'était pas un médecin, mais un orateur professionnel, maître de son art lui aussi, qui prononça ce discours lors d'une occasion solennelle dans le sein de l'Ecole hippocratique de Cos. Discours destiné à glorifier l'art médical; mais il est évident que l'orateur s'est vu obligé de défendre expressément la médecine contre les attaques auxquelles elle était exposée dans la société d'alors. Il le fait avec sagacité avec tant de sagacité que l'on se demande s'il croit lui-même tout ce qu'il dit. Cependant, en sa qualité d'orateur, de sophiste, il n'était point obligé de le croire...

Plus réaliste que le panégyrique «De arte» est le petit texte dit «La Loi» (Lex, Nomos) dejà cité. Ici, c'est bien un médecin expérimenté qui parle. Il sait qu'il existe de bons médecins et de mauvais; il regrette que le public ne sache pas distinguer les uns des autres, ce qui a pour conséquence fâcheuse 
que la profession tout entière tombe en discrédit. La raison qu'il donne pour cet état de choses, c'est l'absence de tout contrôle de l'exercice de la médecine par les autorités des cités grecques. Mais chose étonnante, voire admirable: c'est exactement dans ce climat de liberté professionnelle absolue, dans l'absence de tout règlement officiel, que s'est développé cet art médical que nous appelons hippocratique, d'après son initiateur et représentant principal.

Vivant et exerçant dans un milieu politique et social où l'Etat ne prenait pas de sanctions contre les guérisseurs non qualifiés et ne donnait pas de protection aux médecins bien instruits, les médecins hippocratiques assuraient leur réputation en prenant leur tâche très au sérieux. Ils exigeaient, de leurs disciples comme d'eux-mêmes, des connaissances approfondies et une morale professionnelle élevée. Aux obligations communes, formulées dans le Serment Hippocratique, correspondait une espèce d'esprit de famille. Il m'est impossible de dire la part qu'y avait le facteur religieux: ils vénéraient tous en Asclépios leur aïeul mythique et le patron divin de tous les vrais médecins?

Après avoir déploré le peu de considération dont jouit la médecine, la «Loi» énumère les conditions qui doivent être remplies pour qu'un jeune homme devienne un bon médecin ${ }^{8}$ :

\footnotetext{
«Disposition naturelle, enseignement, lieu favorable [pour ces études], instruction dès l'enfance, amour du travail, longue application.» - «Voila donc les conditions qu'il importe de réunir pour étudier la médecine, voilà la connaissance approfondie qu'il faut en acquérir, si l'on veut, parcourant les villes [pour y pratiquer], être réputé non seulement médecin de nom, mais encore médecin de fait.»
}

\section{Médecins itinérants et médecins publics}

Les paroles que je viens de citer nous rappellent que la vie du médecin hippocratique se développait, en général, d'une autre manière que la carrière d'un médecin de nos jours: il était normal qu'il se déplace souvent, qu'il passe, après quelque temps, d'une ville à l'autre. Le médecin grec était souvent un médecin itinérant. C'est avec son long bâton et assis sur sa chaise portative qu'une stèle funéraire, sculptée vers 480 av. J.-C. et exposée maintenant au Antikenmuseum de Bâle, nous montre le médecin défunt, et c'est aussi le bâton du médecin itinérant qui est entré dans le symbole de la profession, le caducée d'Asclépios. 
Les migrations d'un médecin n'étaient pas toujours complètement volontaires, comme le montre l'histoire de Démocède de Crotone en Italie, racontée par Hérodote ${ }^{9}$. Après s'être brouillé avec son père, Démocède s'en alla en Grèce et pratiqua la médecine à Egine. Il y devint médecin public, mais l'année suivante, les Athéniens l'attirèrent dans leur cité en lui offrant un salaire plus élevé. Le tyran Polycrate de Samos lui offrait encore plus, mais après la chute de ce roi infortuné (en 522 av. J.-C.) Démocède tomba dans les mains du roi de Perse, Darius, et il lui fallut beaucoup de chance et de ruse pour regagner sa liberté et sa patrie. Cela se passait au $6^{\mathrm{e}}$ siècle, donc à l'époque préhippocratique. La tradition itinérante, chez les médecins grecs, était donc ancienne, et elle continua jusque dans l'Empire Romain.

Le récit que donne, au $5^{\mathrm{e}}$ siècle, Hérodote de l'étonnante vie de Démocède nous montre aussi que la fonction du médecin de ville, du médecin public, était pour lui, Hérodote, une institution déjà traditionnelle. En effet, cette fonction représente la seule situation stable qu'un médecin pouvait trouver dans les cités grecques soit à l'âge classique, soit à l'époque hellénistique.

Les idées qu'on se fait du médecin public des cités grecques, du iatros dêmosieuôn, diffèrent considérablement. Les uns veulent y voir une anticipation d'un secteur de la Sécurité Sociale de nos jours: un service médical gratuit aux frais du trésor public - gratuit pour tout le monde ou, au moins, pour la population moins aisée. Mais cela n'est qu'une projection dans le passé de conceptions modernes. D'après les études approfondies de CohnHaft ${ }^{10}$, de Kudlien ${ }^{11}$ et de Nutton ${ }^{12}$, le contrat conclu par la cité avec son médecin public n'avait pour but que d'assurer aux habitants de la ville la présence permanente d'un médecin qualifié pour une période déterminée, en général une année. Cela était particulièrement important dans le cas d'une épidémie grave. Pour son engagement, le médecin touchait un salaire, mais il avait le droit de percevoir des honoraires de ses patients. S'il n'en demandait pas aux gens moins fortunés, c'était, de sa part, une générosité qui fut appréciée par les citoyens et qui souvent fut spécialement mentionnée dans les éloges dont nombre de ces médecins furent honorés. Naturellement, le contrat du médecin public était susceptible d'être renouvelé; Nutton cite l'exemple du médecin Ménocrite de Samos qui restait plus de vingt ans au service de la cité de Karpathos.

Ce système du iatros dêmosieuôn avait ses mérites, et une institution tout à fait semblable existe encore de nos jours dans les vallées éloignées des Grisons et du Tessin, où les communes ou les caisses-maladie versent à un médecin qui veut bien s'installer chez eux un salaire fixe de base. 


\section{Les différentes catégories du personnel médical}

Owsei Temkin nous a montré qu'il y a lieu de distinguer, dans la réalité de la vie grecque à partir du temps d'Hippocrate, deux types de médecins ${ }^{13}$ : le simple praticien routinier, qui se contente d'appliquer les connaissances et les tours de main qu'il s'est acquis au cours de son apprentissage, et le médecin instruit, qui sait donner des raisons valables pour tout ce qu'il fait, parce qu'il connaît et comprend la nature de l'homme, les signes des maladies et les vertus naturelles des remèdes. Voici le véritable homme de l'art (technitês) celui que Platon distingue du simple empirique ${ }^{14}$. La réalité de la vie grecque était encore plus variée. Parmi les personnes qui s'occupaient de la santé des autres, il y avait:

- Les vendeurs de plantes médicinales (pharmakopôlai).

- Les mâ̂tres de gymnastique et les masseurs, traitant les blessures des athlètes et leur prescrivant, pour leur entrainement, des régimes supposés salutaires.

- Les sages-femmes, comme Phénarété, mère de Socrate ${ }^{15}$; souvent, elles étaient consultées par les autres femmes pour des troubles de santé quelconques ainsi que pour les maladies des jeunes enfants; de la sorte, une sage-femme pouvait s'acquérir à peu près l'expérience et la renommée d'une praticienne généraliste. Il existe plusieurs stèles funéraires sur lesquelles la défunte est qualifiée de iatria - femme-médecin, dottoressa.

- Les lithotomistes, mentionnés dans le Serment.

- Enfin les magiciens et expiateurs, attaqués par Hippocrate dans le traité «De la maladie sacrée», et d'autres charlatans.

Toutes ses personnes étaient libres de pratiquer une espèce de médecine, leur art de guérir à eux.

Il est évident que le médecin instruit, le maître de l'art, est celui qui se tient dans la tradition d'Hippocrate, tandis que tout le reste se rapproche d'un empirisme plus ou moins net. Donc, les deux types de médecin définis par Temkin se retrouvent dans la réalité sociale des cités grecques, et Kudlien ${ }^{16}$ a constaté leur existence aussi pour les époques hellénistique et romaine.

Aristote les désigne par les noms de dêmiourgos pour le praticien ordinaire et de architektonikos pour le maître de son art - celui qui a une conception rationelle, une connaissance cohérente de la médecine. Il y ajoute un troisième type de «médecin»: celui qui s'est instruit dans cet art comme 
partie de sa culture générale (ho pepaideumenos peri tên technên) ${ }^{17}$. Ce type d'homme cultivé qui connaît la médecine sans en faire sa profession trouvera sa personnification la plus illustre quelques siècles plus tard dans la personne de l'encyclopédiste romain Aulus Cornelius Celsus.

Enfin, chose plutôt étonnante, le médecin empirique peut même être l'esclave d'un médecin instruit ${ }^{18}$ :

«Platon, dans les Lois ${ }^{19}$, nous assure qu'il existe deux sortes de médecins; les uns au sens strict, formés scientifiquement, citoyens libres, qui soignent habituellement des personnes libres; les autres, aides de ceux-là, mais qu'on appelle aussi médecins, libres ou esclaves, qui n'ont qu'une formation empirique et qui traitent d'ordinaire les esclaves: médecine beaucoup plus expéditive que la première, laquelle s'informe patiemment du cas individuel et veut persuader le malade, l'éclairer et le mettre dans des dispositions psychologiques favorables.»

Il ressort du récit de Platon que, dans les cités grecques, la bonne médecine, la médecine hippocratique, n'était pas toujours à la portée de tout le monde! La fonction du médecin public, dont il a été question plus haut, avait été crée justement dans le but de la rendre un peu plus accessible.

\section{Le médecin auprès du malade}

J'aimerais parler encore de l'activité quotidienne des médecin grees - nous voulons observer le médecin hippocratique au lit du malade ${ }^{20}$. Je m'en tiendrai à ces petits traités de la Collection Hippocratique qui s'occupent de la tenue et du comportement du médecin au lit du malade: «Du médecin», «Préceptes» et surtout «De la bienséance». Avec ce choix de textes, nous nous éloignons, il est vrai, du temps d'Hippocrate, qui vécut de 460 à 377/370. Diller place «Du médecin» (Peri iêtrou) au $4^{\mathrm{e}}$ siècle, mais plutôt dans sa deuxième moitié ${ }^{21}$. Le petit traité «De la bienséance» (Peri euschêmosunês, De decenti habitu) fut composé d'après Jones ${ }^{22}$ en tout cas après 300 av. J.-C., et Diller pense même qu'il ne fut écrit qu'au $1^{\mathrm{er}}$ ou au $2^{\mathrm{e}}$ siècle de notre ère; il en serait de même pour les «Préceptes» (Parangeliai) ${ }^{23}$. Par conséquent, «De la bienséance» ne dépeint pas le médecin grec de l'âge classique, mais celui de l'époque hellénistique ou même des premiers siècles de l'Empire romain. Dans un sens plus général, c'est tout de même un médecin hippocratique, parce que c'est la tradition de l'école de Cos qui détermine sa conception de la profession médicale, son sens de la responsabilité vis-à-vis de son art comme à l'égard de ses malades. En exerçant son 
métier, le médecin entre continuellement en contact avec beaucoup de gens; il a besoin de la confiance des autres, et son existence professionnelle dépend largement de sa réputation. Il ne saurait donc être indifférent à l'impression qu'il fait, par sa tenue et ses manières, sur ceux qui s'adressent à lui. Cela doit être particulièrement vrai pour un peuple dont le sens esthétique est aussi développé qu'il l'était chez les anciens Grecs, mais je crois que chez nous aussi les gens regardent souvent l'aspect extérieur du médecin d'un œil plus critique qu'il ne le pense.

Le premier chapitre du petit traité «Du médecin» contient un portrait idéal du médecin hippocratique, dans toute sa dignité ${ }^{24}$; Diller pense, toutefois, que cette image du bon médecin ne diffère guère de l'idéal du bon Athénien de la $2^{\mathrm{e}}$ moitié du $4^{\mathrm{e}}$ siècle av. J.-C.

«Le médecin doit avoir de l'autorité. Il aura une bonne couleur et de l'embonpoint, suivant ce que comporte sa nature; car la foule s'imagine que ceux dont le corps n'est pas ainsi en bon état ne sauraient soigner convenablement les autres. Puis il sera d'une grande propreté sur sa personne; mise décente, parfums agréables, à l'odeur discrète (Littré: dont l'odeur n'ait rien de suspect); car, en général, tout cela plaît aux malades, et il faut y faire attention.

Quant au moral, il sera plein de modération, non seulement réservé dans ce qu'il dit, mais aussi parfaitement régulier dans sa vie; cela fait le plus grand bien à la réputation. Ses mœurs seront honorables et irréprochables, et avec cela, il sera pour tous grave, humain, équitable; car l'empressement précipité excite le mépris, quand même il serait tout à fait utile. $[\ldots]$

Il aura la physionomie réfléchie, sans austérité; autrement il paraîtrait arrogant et misanthrope. D'un autre côté, celui qui se laisse aller au rire et à une gaieté excessive est regardé comme vulgaire, et cela, il faut s'en préserver soigneusement.»

Il ressort de ce texte qu'il y a, dans la conception hippocratique du médecin, correspondance parfaite entre l'extérieur et l'intérieur; le médecin tel qu'il est dessiné ici est, pour ainsi dire, la personnification de l'art médical, cette iatrikê technê fondée sur des connaissances solides, sûre de ses résultats dans certaines limites, honnête dans ses intentions, donc digne de confiance à tout égard.

Les chapitres 7-17 de la «Bienséance» - texte nettement plus tardif nous permettent de suivre notre confrère distingué quand il se rend chez un malade ${ }^{25}$. Encore une fois, l'auteur insiste sur la tenue et l'attitude du médecin (ch.7): Qu'il ne découvre pas trop, ni de son corps ni de ses réflexions! qu'il se borne, dans ses explications envers les profanes, à ce qui est vraiment nécessaire! Qu'il montre, toutefois, 
«une certaine urbanité; car la rudesse repousse et les gens en santé et les gens malades»!

Qu'il ne fasse

«rien qui soit entaché de recherche ou d'ostentation»!

Voici un des grands principes de la pratique hippocratique, et dont je suis persuadé qu'il découle de l'enseignement d'Hippocrate même. C'est surtout dans les traités chirurgicaux de la Collection Hippocratique que ce principe salutaire est énoncé et illustré à plusieurs reprises ${ }^{26}$.

N'insistons pas sur les préparatifs au domicile du médecin, dans son iatreion: la préparation des médicaments et des instruments, de la trousse portative pour les voyages $(\mathrm{ch} .8,10)$ ! La chose la plus nécessaire à emporter, c'est ce que le médecin doit avoir fixé fermement dans sa mémoire: la connaissance des méthodes de traitement et des actions des médicaments (ch.9):

«C'est là, en médecine, le commencement, le milieu et la fin.»

Bien préparé, intellectuellement, moralement et matériellement, le médecin se met en route, et maintenant, notre auteur exige de lui une chose vraiment extraordinaire (ch. 11):

«Sachez ce qui est à faire avant d'entrer; car beaucoup de cas ont besoin non de raisonnement, mais d'intervention secourable».

Dès qu'il arrive au lit du malade, le médecin doit se montrer maître de la situation! Tout de suite, il énoncera un pronostic:

«cela augmente la réputation et s'apprend facilement».

Le pronostic a donc gardé toute son importance pour le médecin de l'époque alexandrine (ou romaine-impériale). Mais tandis que pour Hippocrate, auteur du «Prognôstikon», le pronostic était le résultat d'un examen attentif du malade et de ses excrétions, l'auteur de la «Bienséance» se montre partisan de la Blitzdiagnose, du diagnostic-pronostic intuitif et immédiat. Il ne prend pas d'anamnèse non plus, ce qui est conforme à la tradition de Cos: l'homme de l'art sait reconnaître l'état d'un malade sans avoir besoin de le questionner; il sait même reconnaître ce que le malade lui cache, soit par négligence, soit délibérément ${ }^{27}$. (Notre médecine contemporaine, sûre de ses techniques de laboratoire et de ses enregistrements électriques, paraît se plaire parfois dans le même mépris de l'anamnèse!) D'autre part, les 
«Préceptes», apparentés à la «Bienséance» a plusieurs égards, préconisent l'utilité de l'interrogatoire du malade et de son entourage ${ }^{28}$ :

«On ne négligera non plus de se renseigner auprès des gens s'il y a quelque apparence que cela serve pour l'occasion du traitement.»

Sans doute, aussi notre médecin modèle-de-bienséance s'est d'abord renseigné un peu sur ce qui l'attend, peut-être en interrogeant habilement le messager qui est venu le chercher; s'il en était autrement, comment pourraitil déjà savoir ce qui est à faire avant même avoir franchi le seuil de la chambre du malade? Malgré l'exhortation à la simplicité que nous avons écoutée (ch. 7), l'apparition de notre médecin au lit du malade n'est, en réalité, pas du tout dépourvue d'ostentation. Au moment de la première rencontre, décisif pour l'établissement d'un bon rapport entre le médecin, le malade et sa famille, les considérations psychologiques l'emportent quelque peu sur les règles sobres et sévères de l'art!

Même en ce moment crucial, le médecin reste conscient de son attitude et de ses mouvements, notamment de «la manière de s'asseoir» (ch. 12). Puis, il concentre son attention et sa sollicitude sur l'homme malade, tout en tenant en respect, par sa dignité, sa réserve et son sang-froid, l'entourage anxieux et excité.

Ayant appliqué et ordonné ce qu'il faut, le médecin prend soin que le malade soit convenablement couché, en tenant compte de la saison et $\mathbf{d u}$ genre de la maladie (ch.15). Si le cas est assez grave et le régime prescrit plutôt compliqué (ce qui arrive facilement), il laisse le patient sous la garde d'un élève suffisamment avancé dans la connaissance de l'art, non seulement pour garantir l'application stricte des mesures thérapeutiques, mais aussi pour y ajouter quelque chose en cas de nécessité (ch.17). En plus:

«il est là aussi afin que rien de ce qui arrive dans l'intervalle des visites ne soit ignoré de vous. Ne vous remettez jamais de rien sur les personnes étrangères à l'art; autrement, le blâme de ce qui sera mal fait retombera sur vous.»

Il est bienséant, voire nécessaire de visiter souvent le malade et de le réexaminer soigneusement lors des visites successives (ch.13). Ainsi, le médecin apercevra à temps tout changement au cours de la maladie et pourra y adapter son traitement.

Hippocrate lui-même avait déclaré ${ }^{29}$ :

«Il est indispensable que s'oppose à la maladie le malade aidé par le médecin.» 
Le médecin, «serviteur de l'art», et le malade sont donc des partenaires, des alliés dans la lutte contre la maladie. Mais le médecin grec, tel que le voit l'auteur plutôt désabusé de la «Bienséance», ne comptait pas trop sur son allié, tourmenté à la fois par les symptômes de la maladie et les inconvénients du traitement médical. Au contraire, il tenait compte de sa faiblesse humaine et s’attendait à la négligence, voire la désobéissance de sa part (ch. 14):

«Il faut observer les fautes des malades; il est arrivé plus d'une fois qu'ils ont menti au sujet des choses prescrites; ne prenant pas les breuvages désagréables, soit purgatifs, soit autres remèdes, ils ont succombé, et le fait ne s'avoue pas, mais l'inculpation est rejetée sur le médecin.»

A qui la faute? au malade ou au médecin? - Nous voici en face d'une querelle apparemment éternelle! En tout cas, c'est une raison de plus pour laisser un élève fidèle et fiable auprès du malade. Celui-ci ne doit, en général, pas s'apercevoir de la méfiance discrète de son grand allié, le médecin. Certes, il est des situations où il faut «le réprimander avec vigueur et sévérité», mais aussitôt, il y aura lieu de nouveau de «le consoler avec attention et bienveillance» (ch.16). Ce qu'il y a de plus important, c'est évidemment que le médecin montre au malade sa sollicitude et qu'il lui inspire du courage. On peut se demander, si, dans nos hôpitaux et policliniques d'aujourd'hui, le précepte suivant est suffisamment respecté (ch. 16):

«On fera toute chose avec calme, avec adresse, cachant au malade, pendant qu'on agit, la plupart des choses, lui donnant avec gaité et sérénité les encouragements qui conviennent.»

\section{Le pronostic néfaste - le principe de la double vérité}

Dans ce contexte, le $16^{\mathrm{e}}$ chapitre de la «Bienséance» touche une difficulté qui, aujourd'hui encore, continue à peser sur les rapports entre médecins et malades. J'ai insisté sur l'importance fondamentale du pronostic pour le médecin grec: sa réputation en dépend. Il a tout intérêt à prononcer clairement aussi un pronostic défavorable, soit pour être disculpé, si son traitement ne réussit pas, soit pour être en droit de refuser ses soins. A la fin du chapitre 17, l'auteur le répète:

«Déclarez donc tous vos pronostics sur les choses que vous faites à ceux qui ont intérêt à les connaître!» 
Mais qui sont ceux qui ont intérêt à connaître, et - suivant la traduction de Jones - à connaître d'une manière plus ample? Le malade lui-même, est il du nombre de ceux que le médecin doit informer complètement? Cela ne paraît pas être l'avis de notre auteur, qui enjoint au médecin (ch.16) de

«ne lui laisser rien apercevoir de ce qui arrivera ni de ce qui menace; car plus d'un malade a été mis à toute extrémité par cette cause, c'est-à-dire par un pronostic où on lui annonçait ce qui devait arriver ou ce qui menaçait.»

«Eph'hetera apeôsthêsan» - «ils ont été mis à toute extrémité», cela veut-il dire que ces malades ont péri dans la désolation, privés de toute force de résistance, ou même qu'ils se sont suicidés? Peu importe: le malade ne supporte pas «la vérité» néfaste telle qu'elle apparaît au médecin. Cela est devenu presqu'un dogme de la psychologie médicale.

«Il faut toujours donner au malade espérance,

encore que de mort y ait grande apparence»,

enseignera Ambroise Paré au $16^{\mathrm{e}}$ siècle $^{30}$.

Mais si nous tournons le regard en arrière, nous constatons que les choses ont bien évolué depuis le «Prognôstikon», écrit par Hippocrate vers 400 av. J.-C., jusqu'à la «Bienséance» de son disciple lointain. Hippocrate enseignait ${ }^{31}$ :

«Il faut connaître la nature de ces maladies [éventuellement mortelles], dans quelle mesure elles sont supérieures aux forces des corps, de même s'il y a en elles quelque chose de divin, et il faut se graver dans la mémoire leur pronostic. Ainsi, on sera admiré à juste titre et on sera un excellent médecin.»

Pour les médecins hippocratiques de la première heure, les médecins des villes de la Grèce classique, le pronostic ne servait donc pas seulement, et pas en premier lieu, à guider la thérapeutique, mais surtout à établir la renommée du médecin auprès du public. Le médecin se faisait respecter comme juge impeccable du destin du malade. Le pronostic infaillible était la marque suprême du savoir médical. Si le malade s'en désolait, s'il n'était pas assez fort pour supporter le verdict médical, la «révélation massue» selon la formule de Danielle Gourévitch ${ }^{32}$, du pronostic néfaste, tant pis; le confrère d'Hippocrate ne s'en sentait pas responsable. En revanche, il se serait senti malhonnête et même coupable s'il avait évoqué de vains espoirs de guérison.

Pour l'auteur de la «Bienséance», qui exerçait peut-être à Alexandrie ou dans une autre ville hellénistique, les choses ne sont plus aussi simples. Le médecin se sent responsable, justement, aussi du moral de ses patients. Donc, 
il cache au malade même le pronostic réaliste et sombre, mais il le révèle à ses proches. Voici ce principe de la double vérité, cette règle pragmatique qui guide le comportement des médecins jusqu'à nos jours. Le principe a été élaboré, à mon avis, pour la première fois dans ce petit livre «Sur la bienséance», qui traduit l'expérience des médecins de tradition hippocratique dans les villes hellénistiques. Par comparaison avec les temps antérieurs, à l'époque d'Hippocrate, la psychologie du malade a conquis une importance plus grande.

Certes, ce principe de la double vérité, vivement contesté aujourd'hui, conseille au médecin un comportement qui n'est point idéal, ni intellectuellement, ni moralement. Mais elle représente souvent le moindre des maux, surtout si l'on prend en considération que la «vérité» d'un pronostic médical est également bien relative.

\section{Notes}

1 D'après une conférence donnée à l'Université de Padoue.

2 Corpus Hippocraticum (CH), Lex, ch. 1, Littré 4, 638/9.

3 C. Plinius Secundus, Historia naturalis 29, ch. 8, §18 - éd. A. Ernout, Paris (Belles Lettres) 1962, 26.

4 Danielle Gourévitch, Le triangle hippocratique dans le monde gréco-romain, Rome (Ecole française) 1984, $2^{\mathrm{e}}$ partie, ch.4: «L'Anti-Hippocrate ou le roman noir de la médecine», 347-414.

5 CH, De arte, ch.4-8, Littré 6, 6-15.

6 Plinius, op. cit. (voir note 3), ch. 7, §14, p. 24.

7 Au sujet des Asclépiades, comparez dans cette revue l'article de Jutta Kollesch sur Cnide, p. 11. - En outre: Emma J.Edelstein and Ludwig Edelstein, Asclepius, a Collection and Interpretation of the Testimonies, 2 vols., Baltimore (Johns Hopkins) 1945 (reprint New York 1975).

«By calling themselves Asclepiads, by considering themselves a unity, a family though not in blood, the physicians formed one of the first corporations of craftsmen.» $(2,60)$.

8 CH, Lex, ch. 2 et 4, Littré 4, 638-641.

9 Herodotus, Historiae 3, ch. 129-137.

10 Louis Cohn-Haft, The Public Physicians of Ancient Greece, Smith College Studies in Hist. 4.2, Northampton, Mass., 1956.

11 Fridolf Kudlien, Der griechische Arzt im Zeitalter des Hellenismus, Akad. Wiss. u. Lit. Mainz, Abhandl. geistes- u. soz.-wiss. Klasse, Jg. 1979, Nr. 6, Wiesbaden (Steiner) 1979.

12 Vivian Nutton, Continuity or Rediscovery? The City Physician in Classical Antiquity and Mediaeval Italy, dans: The Town and State Physician in Europe [...], ed. by Andrew W. Russell, Wolfenbüttel (Herzog August Bibl.), Wolfenbütteler Forschungen 17, 1981, $9-46$. 
13 Owsei Temkin, Greek Medicine as Science and Craft, Isis 44 (1953), 213-225. Trad. allemande: Griech. Medizin als Wissenschaft und Handwerk, dans: Antike Medizin, herausg. von Hellmut Flashar, Darmstadt (Wiss. Buchges.), Wege der Forschung CCXXI, 1971, 1-28.

14. Plato, Gorgias, 4.65 A.

15 Plato, Theaetetus, $149 \mathrm{C} / \mathrm{D}$.

16 Kudlien, op. cit. (voir note 11).

17 Aristoteles, Politica 3, ch.11, 1282 a.

18 Robert Joly, Esclaves et médecins dans la Grèce antique, Sudhoffs Archiv 53 (1969), 1-14. Citation des pp. $1 / 2$.

19 Plato, Leges 4, 720 A-E; 9, 857 C/D.

20 Voir aussi: Huldrych M. Koelbing, Le médecin hippocratique au lit du malade, dans: Hippocratica, Actes du colloque hippocratique de Paris (1978), éd. par Mirko D. Grmek, Paris (CNRS) 1980, 321-331.

21 Hans Diller, dans: Hippokrates, Schriften, Reinbek/Hamburg (Rowohlts Klassiker 108/109) 1962, 87.

22 W. H.S.Jones, Hippocrates 2, Cambridge, Mass./London (Harvard Univ./Heinemann), Loeb Class. Libr., 1923 etc., 271.

23 Diller (voir note 21), 95. - Jeanne Ducatillon place les «Préceptes» au $1^{\text {er }}$ s. av. J.-C.: Le médecin opsimathe d'après le ch. 13 du traité des Préceptes, dans: Hippocratica (voir note 20), 113-133.

24 CH, Medicus, ch.1, Littré 9, 204-207; Walter Müri, Der Arzt im Altertum, $3^{e}$ éd., München (Heimeran) 1962 (réédité depuis par Artemis, Zürich/München), 20-23, d'après CMG 1,1 .

25 CH, De decenti habitu, Littré 9, 236-243; Jones (voir note 22), 290-301; Müri (voir note 24), 28-31.

26 Voir Huldrych M.Koelbing, Arzt und Patient in der antiken Welt, Zürich/München (Artemis) 1977, 100/101.

27 CH, Regimen in acutis, ch. 1; Prognosticum, Ch. 1.

28 CH, Praecepta, ch.2, Littré 9, 254/5.

29 CH, Epidem. 1, ch. 11.

30 Ambroise Paré, Canons et reigles chirurgiques, dans: Euvres complètes, éd. par. J.F. Malgaigne, tome 3, Paris (Baillière) 1841, 650 (canon 38).

31 CH, Prognosticum, ch. 1, trad. personnelle.

32 Danielle Gourévitch, Déontologie médicale: quelques problèmes, dans: Mélanges d'archéol. et d'hist. (Rome, Ec. franç.) 81 (1969), 519-536, et 82 (1970), 737-752.

\section{Summary}

\section{The physician in the Greek city}

In the cities of ancient Greece, as well as later in Rome, the doctor's responsibility was already a controversial subject. The practice of healing was not subject to any official regulation: no protection of good physicians, no punishment of malpractice. While physicians often lead an 
itinerant life, cities endeavoured to secure the presence of a good one by appointing him town or public physician on the basis of a one-year contract. This did not mean, however, a "health service" free of charge for patients. - The variety of healing persons including midwives and medical slaves is reviewed.

Some short texts which were added in later times to the "Works of Hippocrates" ("Physician", "Precepts", "Decorum") provide us with some information on a physician's daily life (see also H.M. Koelbing, The Hippocratic physician at his patient's bedside, in Practitioner 224, 1980, 551-554). From Hippocrates ("Prognostic") to the hellenistic period ("Decorum"), we note an important change as to the revelation of a bad prognosis: Hippocrates advocates the blunt information of the patient when there is no hope for him; but his follower in a later century takes into consideration the patient's psychology. He hides the cruel truth from him while informing openly his relatives and near friends. This is the first time in history we come across the principle of the doctor's double truth, strongly, advocated e.g. by Thomas Percival in his "Medical Ethics" (1803), but much disputed today.

\section{Zusammenfassung}

\section{Der Arzt in der griechischen Polis}

In den griechischen Städten wie später in Rom war die Verantwortung des Arztes bereits ein Gegenstand der Auseinandersetzung. Die Heiltätigkeit war ein vollkommen freies Gewerbe: kein staatlicher Schutz für den gut ausgebildeten, verantwortungsbewußten Arzt - keine Sanktionen gegen Scharlatane. Auch tüchtige Ärzte führten oft ein Wanderleben; aber die Städte suchten sich durch die Ernennung von Stadtärzten (die jedoch nicht zu unentgeltlicher Behandlung verpflichtet waren), die Dienste qualifizierter Ärzte auf Zeit zu sichern. Die guten Ärzte, d.h. die Ärzte nach hippokratischem Vorbild, sahen sich der Konkurrenz einer Vielzahl bloß empirisch geschulter Praktiker (incl. Hebammen) gegenüber; einige ließen auch einen Teil ihrer Klientel durch eigene Sklaven behandeln, wie wir von Platon wissen.

Einige kurze, in späterer Zeit dem Corpus Hippocraticum beigefügte Texte vermitteln uns einen Einblick in den Alltag des griechischen Arztes. Von Hippokrates («Prognôstikon») zum Hellenismus («De decenti habitu» - «Vom Anstand») können wir eine wichtige Wandlung bezüglich der Mitteilung einer hoffnungslosen Prognose feststellen: Während Hippokrates ihre unumwundene Bekanntgabe empfiehlt, trägt in einem späteren Jahrhundert sein entfernter Schüler der Psychologie des Kranken Rechnung; er erspart ihm die niederschmetternde Eröffnung des drohenden Todes, klärt jedoch die ihm Nahestehenden auf. Hier wird erstmals das Prinzip der doppelten Wahrheit des Arztes greifbar, das heute so umstritten ist.

Prof. Dr. med. Huldrych M. Koelbing

Loostraße 19

CH-8803 Rüschlikon ZH 